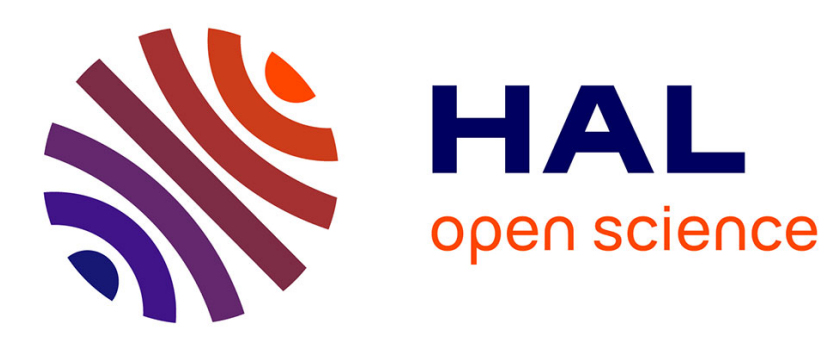

\title{
Combining ARF and OR-PCA for Robust Background Subtraction of Noisy Videos
}

Sajid Javed, Thierry Bouwmans, Soon Ki Jung

\section{To cite this version:}

Sajid Javed, Thierry Bouwmans, Soon Ki Jung. Combining ARF and OR-PCA for Robust Background Subtraction of Noisy Videos. International Conference in Image Analysis and Applications, ICIAP 2015, Sep 2015, Genova, Italy. pp.340 - 351, 10.1007/978-3-319-23234-8_32 . hal-01374219

\section{HAL Id: hal-01374219 \\ https://hal.science/hal-01374219}

Submitted on 30 Sep 2016

HAL is a multi-disciplinary open access archive for the deposit and dissemination of scientific research documents, whether they are published or not. The documents may come from teaching and research institutions in France or abroad, or from public or private research centers.
L'archive ouverte pluridisciplinaire HAL, est destinée au dépôt et à la diffusion de documents scientifiques de niveau recherche, publiés ou non, émanant des établissements d'enseignement et de recherche français ou étrangers, des laboratoires publics ou privés. 


\title{
Combining ARF and OR-PCA for Robust Background Subtraction of Noisy Videos
}

\author{
Sajid Javed ${ }^{1}$, Thierry Bouwmans ${ }^{2}$, and Soon Ki Jung ${ }^{1}$ \\ ${ }^{1}$ School of Computer Science and Engineering, Kyungpook National University, \\ 80 Daehak-ro, Buk-gu,Daegu, 702-701, Republic of Korea \\ \{sajid@vr.knu.ac.kr $\},\{$ skjung@knu.ac.kr $\}$ \\ ${ }^{2}$ Laboratoire MIA (Mathematiques, Image et Applications)- Université de La \\ Rochelle, 17000, France, \{thierry.bouwmans@univ-lr.fr $\}$
}

\begin{abstract}
Background subtraction is a fundamental pre-processing step for many computer vision applications. In addition to cope with dynamic background scenes, bad weather conditions such as rainy or snowy environments and global illumination conditions such as light switch on/off are still major challenging problems. Traditional state of the art methods, such as Robust Principal Component Analysis fail to deliver promising results under these worst conditions. This is due to the lack of global preprocessing or post-processing steps, incorrect low-dimensional subspace basis called low-rank matrix estimation, and memory or computational complexities for processing high dimensional data and hence the system does not perform an accurate foreground segmentation. To handle these challenges, this paper presents an input video denoising strategy to cope noisy videos in rainy or snowy conditions. A real time Active Random Field constraint is exploited using probabilistic spatial neighborhood system for image denoising. After that, Online Robust Principal Component Analysis is used to separate the low-rank and sparse component from denoised frames. In addition, a color transfer function is employed between the low-rank and the denoised image for handling abruptly changing lighting conditions, which is a very useful technique for surveillance agents to handle the night time videos. Experimental evaluations, under bad weather conditions using two challenging datasets such as I-LIDS and Change Detection 2014, demonstrate the effectiveness of the proposed method as compared to the existing approaches.
\end{abstract}

\section{Introduction}

Video background modeling and subtraction is a very crucial step in many image processing applications such as video registration, inpainting, compression and segmentation [18]. This pre-processing step consists of segmenting the moving foreground objects from the static scene called "background". But existence of undesirable weather conditions such as rain, fog, snow or haze is still major challenge for many applications, in addition to bootstrapping and dynamic background subtraction issues, which may cause performance problems in visual surveillance systems. 
A number of interesting frameworks have been developed to tackle the problems of background subtraction in videos [3] and several implementations are available in $\mathrm{BGS}^{1}$ and $\mathrm{LRS}^{2}$ libraries. Robust Principal Component Analysis (RPCA) based low-rank matrix decomposition algorithms using Principal Component Pursuit (PCP) provide encouraging performance for background/foreground separation [3]. RPCA decomposes the original data matrix, as a sum of lowdimensional subspace having intrinsic structure called low-rank matrix (corresponds to the background) and correlated outliers called sparse component (constitutes the foreground objects). For example, $1^{\text {st }}$ row in Fig. 1 shows an example of background subtraction using RPCA of original image taken from I-LIDS dataset [4].

However, due to the batch optimization processing and partial SVD computation at each major loop, RPCA suffers from memory and computational complexities and hence the low-rank matrix can not be estimated correctly due to the lack of constraints. Moreover, earlier RPCA methods also do not provide satisfactory performance under bad weather conditions as depicted in the $2^{\text {nd }}$ and $3^{\text {rd }}$ rows of Fig. 1 of sequences taken from I-LIDS [4] and Change Detection (CDnet) 2014 dataset [7].

In order to tackle these challenges, this paper presents a robust background subtraction algorithm via Online Robust PCA (OR-PCA) on denoised video frames (noise free). We briefly explain our methodology here. First, the continuous constraints such as Active Random Field (ARF) based on the combination of Markov Random Field (MRF) and Conditional Random Field (CRF), are employed on noisy or rainy video frames. Then, OR-PCA is applied on denoised images for background modeling. Since ARF based image denoising technique provides encourging results against noisy pixels. Therefore, our methodology improves the quality of foreground via OR-PCA under mild weather environments. Moreover, without detecting the global illumination conditions, a color transfer function is used between the source and input image to maintain the abruptly changing lighting conditions of current frame when the light switch is turned off. It can be a useful functionality for surveillance agents to select the color transfer option for night time surveillance or illumination changing conditions. Finally, a very nice comparison of ARF based background subtraction with other methodologies is presented in detail.

The rest of this paper is organized as follows. In Section 2, the related work is reviewed. Section 3 describes our methodology in detail. Experimental results are discussed in Section 4. Finally, conclusions are drawn in Section 5.

\section{Related Work}

In the literature, a number of encouraging methods have been proposed for robust background subtraction. Due to the over growing demand of processing high dimensional data, subspace learning models such as RPCA [5] attract a

\footnotetext{
${ }^{1}$ https://github.com/andrewssobral/bgslibrary

${ }^{2}$ https://github.com/andrewssobral/lrslibrary
} 


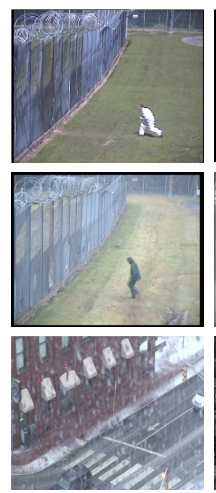

(a)

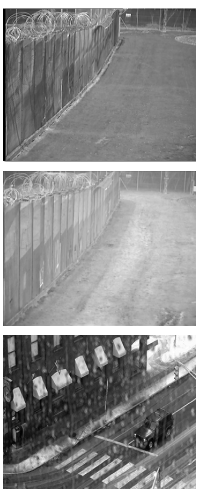

(b)

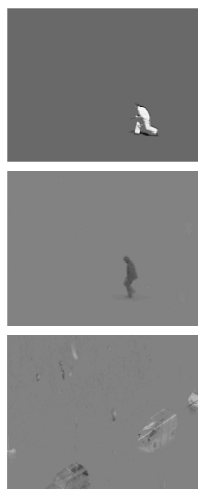

(c)

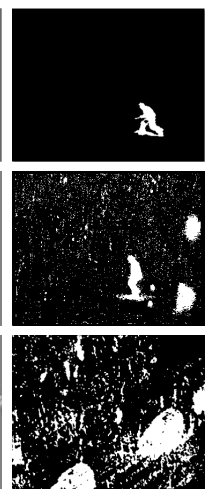

(d)

Fig. 1. An example of background subtraction using RPCA. From left to right: (a) input, (b) low-rank, (c) sparse component, and (d) foreground mask. From top to bottom: non-noisy, rainy, and snowy scene.

lot of attention. Excellent surveys on background modeling using RPCA can be found in [3]. All these RPCA approaches discussed in [3] work according to batch optimization and therefore they are not applicable for real-time systems.

In contrast, Feng and $\mathrm{Xu}[6]$ proposed OR-PCA that alleviates most of the ealier RPCA limitations. OR-PCA processes one frame per time instance via online (also called iterative or stochastic) optimization. In [6], it is argued that OR-PCA converges to the optimal solution and achieves the comparative performance as compared to its batch counterparts. However, no encouraging results over video background subtraction are reported. Therefore, S. Javed et. al [11] modified OR-PCA [6] for background/foreground segmentation. A number of encouraging results are presented in [11]. But annoying parameters tuning is the main drawback in their approach.

All these RPCA methods discussed above perform the low-rank recovery which is robust to sparse corruptions but it gets fail to handle worst weather conditions such as rainy or snowy. To address rain removal from videos using RPCA, A. Hakim [8] proposed a novel framework for rainy video restoration using exact low-rank recovery, but the system performance was degraded as the hard constraints were applied in the low-rank part, which is not useful for online processing. In this study, we propose an integrated framework based on ARF for image denoising, and OR-PCA along with color transfer scheme for robust background subtraction of noisy image sequences.

\section{Proposed Approach}

In this section, we discuss our scheme for background subtraction of noisy videos in detail. Our methodology consists of several components which are described as a system diagram in Fig. 2. 


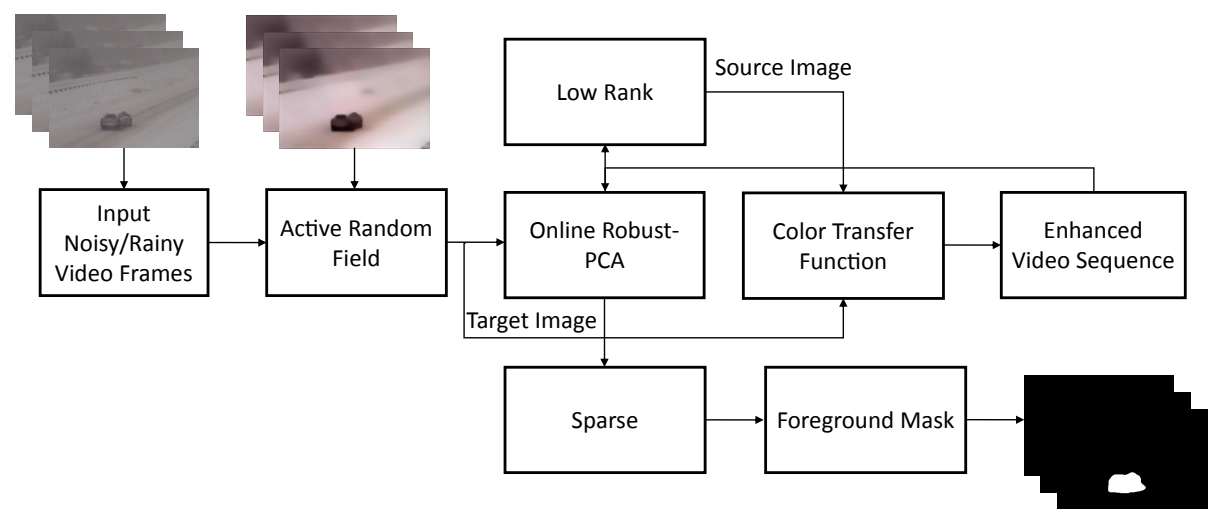

Fig. 2. Block diagram of our proposed scheme.

Our methodology consists of three main stages: ARF, background subtraction, and color transfer function. In this work, instead of applying any mild constraints on low-rank component, we first denoise the input video frames using the continuous ARF, and then, OR-PCA [11] is applied on filtered frames to get the improved foreground segmentation. To handle the global illumination conditions, we use the color transfer scheme for illumination stability without detecting the global changes in a scene. We propose an integrated framework for both bad weather conditions and night time surveillance or abruptly changing lighting conditions. One very useful benefit of our approach is that, the surveillance agent is able to select the color transfer function for night time surveillance. In addition, a very effective comparison is presented in detail with earlier methods using ARF based initialization, and we show that ARF with OR-PCA provides the best results. In the following sections, we will describe each module in detail.

\subsection{Real Time Acive Random Field (ARF) for Image Denoising}

In this work, the rain/snow filtering problem is analyzed using input video restoration method. The original video frame, say $Z_{t}$, is assumed to be three dimensional e.g., width $\times$ height $\times$ color, at a time $t$. This noise free input frame is then corrupted by some noise (rain/snow drops) represented by $y_{t}$. The main goal is to restore this original frame using the continuous constraints like ARF, and then, the background subtraction is performed for improved foreground detection.

The ARF $[1,2]$ model is a combination of probabilistic MRF/CRF model which shows a very nice potential for many computer vision applications such as image segmentation, denoising and stereo disparity using the Fields of Experts MRF model [15] and simple gradient descent inference algorithm. In [1], a very interesting real time image denoising application is proposed. 
The main idea of ARF model is that, through an appropriate training of $\mathrm{MRF} / \mathrm{CRF}$ prior model, and a fast inference algorithm with an optimization of loss function on a given image, the model perform very well for image denoising on a suboptimal inference algorithm. In this work, rainy video is denoised or restored by exploiting the ARF information with the same training data used in $[1,2]$.

Let each pixel be a node in a directed graph $G=(V, E)$, where a set of vertices $V$ stands for all pixels in the image and a set of edges $E$ denotes 4connected spatially neighboring pixels. Let $C$ be a set of cliques of $G$ which is fully connected subgraphs. Then, the main goal is to recover an image $x_{t}$ which is both smooth and close to $y_{t}$ using the gradient descent having energy minimization function given by

$$
E_{x_{t}}=E_{\text {data }}\left(x_{t}\right)+E_{F O E}\left(x_{t}, \theta\right),
$$

where the term $E_{\text {data }}\left(x_{t}\right)$ is a cost function for assigning labels to $x_{t}$ and is given by

$$
E_{\text {data }}\left(x_{t}\right)=\frac{1}{2 \sigma^{2}} \sum_{j}\left(y_{t}^{j}-x_{t}^{j}\right)^{2},
$$

where $x_{t}^{j}$ and $y_{t}^{j}$ is the value of pixel $j$ of image $x_{t}$ and $y_{t}$, and $\sigma$ is a smoothing factor. Similarly $E_{F O E}($ data $)$ is the energy of Fields of Experts which is an MRF prior model having convolution kernels called filters as $J_{f}, f=1, \ldots, N$ with corresponding coefficients $\alpha_{f}$, which is given by

$$
E_{F O E}\left(x_{t}, \theta\right)=\sum_{j} \sum_{f=1}^{N} \alpha_{f} \log \left(1+\frac{1}{2}\left(J_{f} x_{t}^{k}\right)^{2}\right),
$$

where $\sum_{j}$ is the sum over the cliques $k$ of the denoised image $x_{t}$ and $x_{t}^{k}$ are the corresponding pixels of clique $k$. Solving (1) takes thousands of iterations, which is really a hard task for real time systems. A. Barbu [1] designed a loss function with its online optimization available in his homepage ${ }^{3}$, and it normally takes less than a second with four iterations to minimize the energy function and hence applicable for real-time processing. Fig. 3 (b) to (f) show the ARF based denoised images taken from CDnet [7] with different values of $\sigma$. This $\sigma$ value in Fig. 3 (b) to (f) makes the smoothed restored images using the same training data used in $[1,2]$.

\subsection{Background Subtraction}

In this paper, OR-PCA [6] is applied on each denoised video frame for robust low-rank and sparse error separation. OR-PCA basically decomposes the nuclear norm of the objective function of the traditional PCP algorithms into an explicit product of two matrices, i.e., the basis and coefficient.

\footnotetext{
${ }^{3}$ http://www.stat.fsu.edu/abarbu/ARF/index.html
} 


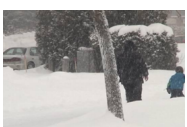

(a)

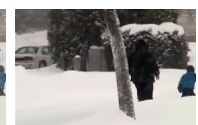

(b)

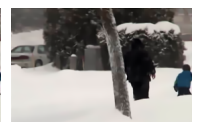

(c)

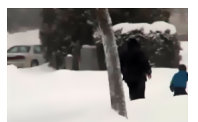

(d)

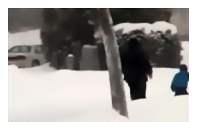

(e)

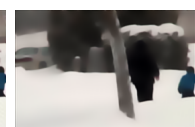

(f)

Fig. 3. ARF based image denoising. From left to right: (a) input, and denoised image using (b) $\sigma=10$, (c) $\sigma=15$, (d) $\sigma=20$, (e) $\sigma=25$, and (f) $\sigma=50$, respectively.

In [6], an iterative or stochastic optimization scheme is designed for OR-PCA and it is proved that the method converges to the global optimal solution of the original PCP formulation. OR-PCA can be formulated as

$$
\begin{aligned}
\min _{L \in \Re^{p \times d}, R \in \Re^{n \times d}, E}\{ & \left\{\frac{1}{2}\left\|D-L R^{T}-E\right\|_{F}^{2}\right. \\
+ & \left.\frac{\lambda_{1}}{2}\left(\|L\|_{F}^{2}+\|R\|_{F}^{2}\right)+\lambda_{2}\|E\|_{1}\right\},
\end{aligned}
$$

where $D$ is the column-vector of denoised images e.g., $D \in \Re^{p \times n}, p$ is the number of pixels with three color features, e.g. (width $\times$ height $\times 3$ ), $n$ denotes the number of samples (video frames), $d$ is a rank, $L$ is the optimum basis of each individual color channel, $R$ is a basis coefficient, and $E$ is a sparse error. $\lambda_{1}$ controls the basis and coefficients for low-rank matrix, whereas $\lambda_{2}$ controls the sparsity pattern, which can be tuned according to video analysis. In addition, basis and coefficients depend on the value of rank $r$, which is tuned carefully to speed up the stochastic optimization process.

In particular, the OR-PCA optimization consists of two iterative updating components. First, every incoming restored image is projected onto current initialized basis $L$ and we separate the sparse noise component, which includes the outliers contamination. Then, the basis $L$ is updated with a new denoised color image individually. More details can be found in [6].

The background sequence for each image is then modeled by a multiple of basis $L$ and its coefficient $R$, e.g., $X=L R^{T}$, whereas the sparse component $E$ for each image constitutes the foreground objects which is then thresholded to get the binary mask using hard thresholding scheme.

\subsection{Color Transfer Method}

The low-rank component is not always consistent and needs to be updated at a time $t$, especially for abruptly changing lighting conditions, such as turning off the light switched in indorr scenes. The surveillance system always show a very weak performance due to rapid lighting variations.

Many approaches have been developed to detect change of global illumination conditions such as in [16]. However, the delay always arise when the new pixels are updated which is the main drawback in earlier approaches. Color transfer between images has shown to be a very efficient strategy, initially developed 
for computer graphics applications $[10,14]$ to transfer the desired colors into an input image.

In this work, we transfer the color between the low-rank and input denoised images, without detecting the intense lighting variations, and this can be done by taking the bright colors from low-rank matrix, and then, transform the color map of the input video sequence. The OR-PCA is then employed on enhanced video sequence for improved foreground segmentation. This color transfer scheme is very important especially for night time surveillance, when a bright day time low-rank scene is computed online using OR-PCA, and then, it is used to transform the colormap of the night time scene for video enhancement. However, we have not stored any bright time low-rank scene in this study, but a series of these images can be stored if not available during night time surveillance, and then, the weighted low-rank component which can be computed using the linear combinations of these images, can be adopted as a source image for color transfer function.

E. Reinhard et. al [14] proposed a very efficient and simple color transfer algorithm using decorrelated color space between the source and target image. Let say that $I_{s}$ and $I_{t}$ be an RGB source (low-rank component obtained from OR-PCA) and target image (current denoised video frame).

The $I_{s}$ and $I_{t}$ images are first converted into $l \alpha \beta$ color space using the formulation presented in [14]. Then, $l_{s} \alpha_{s} \beta_{s}$ and $l_{t} \alpha_{t} \beta_{t}$ are each source and target $l \alpha \beta$ color spaces after conversion. Next, the mean value is subtracted from each individual axis and standard deviation is divided from the source image with a multiple of enahanced source $l \alpha \beta$ axis. The standard deviation of each $I_{t}$ axis is added separately, and finally the enhanced $l^{\prime} \alpha^{\prime} \beta^{\prime}$ space is then converted back into the RGB color space after the color transfer function given by

$$
l^{\prime}=\frac{\sigma_{t}^{l}}{\sigma_{s}^{l}} l^{*}+\mu_{l, t}, \alpha^{\prime}=\frac{\sigma_{t}^{\alpha}}{\sigma_{s}^{\alpha}} \alpha^{*}+\mu_{\alpha, t}, \beta^{\prime}=\frac{\sigma_{t}^{\beta}}{\sigma_{s}^{\beta}} \beta^{*}+\mu_{\beta, t}
$$

where $l^{*}=l_{s}-\mu_{l, s}, \alpha^{*}=\alpha_{s}-\mu_{\alpha, s}$ and $\beta^{*}=\beta_{s}-\mu_{\beta, s}$. Similary the $\mu$ and $\sigma$ are the mean value and standard deviations of each source and target $l \alpha \beta$ axis in (5). More details can be found in [14]. Fig. 4 (a) to (e) show the enhanced video results using $A R F$ with color transfer scheme for improved background subtraction. The average RGB pixel is also computed for evaluation purpose and Fig. 4 (f) depicts that the color transfer video has more stable illumination condition.

\section{Experimental Evaluations}

In this section, experimental results are reported for bad weather conditions and night videos category, taken from the two well-known challenging datasets such as CDnet [7] 2014 and I-LIDS [4] dataset.

We have also evaluated and studied several state of the art approaches by integrating ARF with Mixture of Gaussians [17] (ARF-MOG), PBAS [9] (ARFPBAS), Codebook [12] (ARF-CB) and some recent methods, e.g., FTSG [19], 

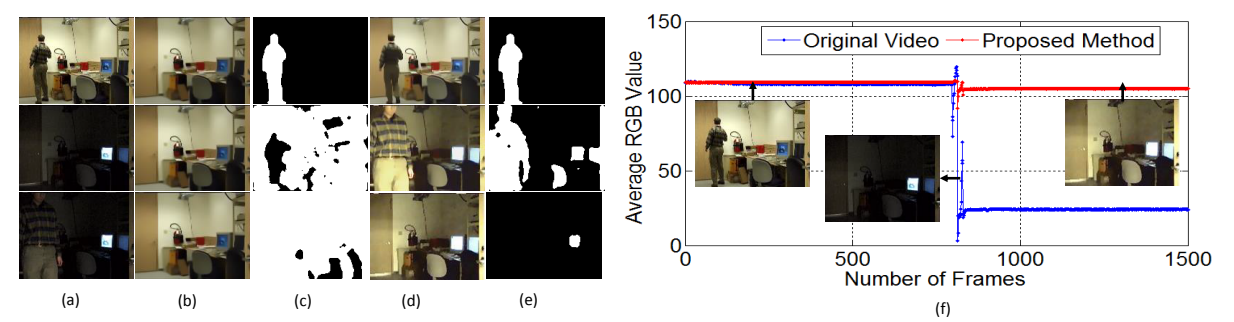

Fig. 4. Color transfer between low-rank and input denoised image. From left to right: (a) input, (b) low-rank, (c) binary mask without color conversion, (d) video sequence using color conversion, (e) foreground mask with color conversion, and (f) comparison of RGB pixel values between original and restored video using color transfer function.

Bin Wang Apr [18], and MSTB model [13] with results publicly available in CDnet $^{4}$. We use the parameters in (4) as $d=6, \lambda_{1}=0.01, \lambda_{2}=0.05$, and $\sigma=20$ for ARF video denoising. First the qualitative results are presented, and then, quantitative study is described in details.

\subsection{Qualitative Results}

The visual results are presented on some selected video sequences from each dataset due to the space limitations. The proposed approach is implemented on Matlab R2013a with $3.40 \mathrm{GHz}$ Intel core i5 processor and 4 GB RAM. Morever, a $5 \times 5$ median filtering is applied on binary mask.

CDnet [7] 2014 is a well-known real-time challenging dataset, which contains the category called Bad weather condition and Night videos. The $1^{\text {st }}$ category contains 4 major video sequences called Blizzard, Skating, Snow fall, and Wet snow. The $2^{\text {nd }}$ one consists of 6 videos, but only two sequences called Bridge entry and Tram station are presented. In this dataset, the Night videos do not contain any day time scene so we directly apply our approach without color transfer sheme. The image size of $1^{\text {st }}$ category is $720 \times 480$, whereas the other videos contain $540 \times 360$ and $720 \times 540$ frame sizes, and half of the resolution is used in our experiments.

We have also tested some non-noisy videos under stable illumination condition from CDnet dataset [7] using the Baseline category. This category contains 4 basic videos namely: Highway, Office, Pedestrians, and PETS2006. These nonnoisy sequences that contain stable lighting condition are also pre-processed using the ARF constraints, and then, the background subtraction is performed. Due to the denoised non-noisy images satisfactory smoothing properties, a small moving pixels are suppressed that eradicates most of the false alarms from the binary mask. However, the color transfer scheme has no affect in this case, since the source image i.e., the low-rank component and denoised non-noisy image contains the same bright scene. Fig. 5 (a) to (d), show the results on Bad Weather

\footnotetext{
${ }^{4}$ http://www.changedetection.net/
} 


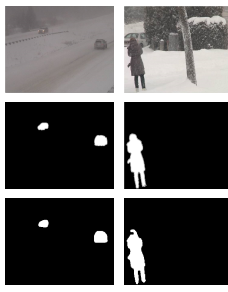

(a)

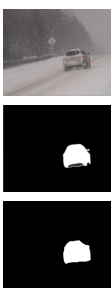

(c)

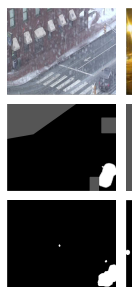

(d)

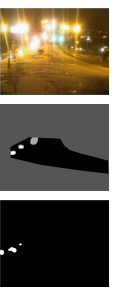

(e)

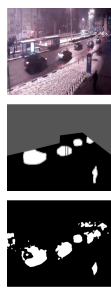

(f)

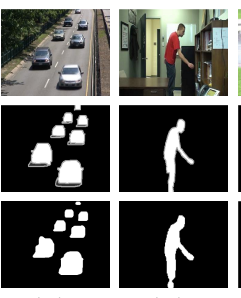

(g)

(h)

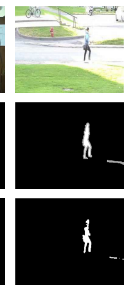

(i)

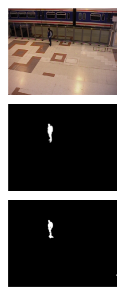

(j)

Fig. 5. Results of the proposed method. From left to right: (a)-(d) Bad Weather videos: (a) Blizzard, (b) Skating, (c) Snow fall, and (d) Wet snow. (e)-(f) Night videos: (e) Bridge entry, and (f) Tram station. (g)-(j) Baseline category: (g) Highway, (h) Office, (i) Pesdestrians, and (j) Pets2006. From top to bottom: input, ground truth, and our results.

Condition videos, whereas the visual results of Night Videos are shown in Fig. 5 (e) to (f). In addition, the results on Baseline video sequences are also reported in Fig. $5(\mathrm{~g})$ to $(\mathrm{j})$.

I-LIDS [4] is the Imagery Library for Intelligent Detection Systems dataset which consists of about 213 video sequences. Among them, the category SZTRA104b contains 10 videos of worst weather environment. The image size of each sequence is $576 \times 720$. Fig. 6 depicts the visual results of 5 sequences using I-LIDS dataset.
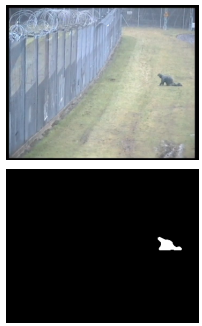

(a)
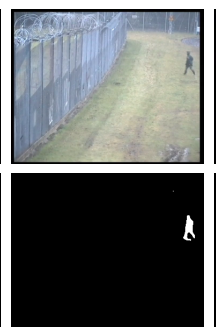

(b)
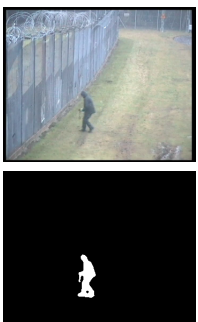

(c)
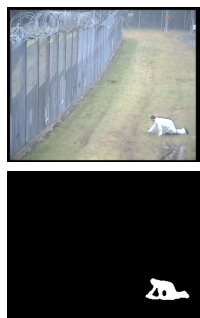

(d)

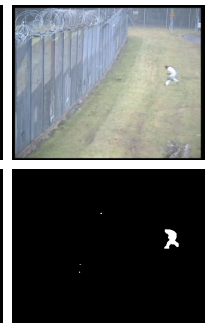

(e)

Fig. 6. I-LIDS category SZTRA104b. From left to right: (a) SZTRA104b01, (b) $S Z$ TRA104b04, (c) SZTRA104b06, (d) SZTRA104b08, and (e) SZTRA104b09. From top to bottom: input and results of our method.

\subsection{Quantitative Results}

For quantitative evaluations, we have computed the F-measure score for all sequences, by comparing our results with their available corresponding ground truth data. The $F$-measure is given as

$$
F_{\text {measure }}=\frac{2 \times \text { Recall } \times \text { Precision }}{\text { Recall }+ \text { Precision }},
$$


where Recall and Precision are computed based on true positives, false posi-

Table 1. Qualitative results of CDnet dataset [7]: Average F-measure score of each video sequence with earlier approaches.

\begin{tabular}{|c|c|c|c|c|c|c|c|c|c|c|c|}
\hline \multirow{3}{*}{ Method } & \multicolumn{8}{|c|}{ Change Detection dataset [7] } \\
\cline { 2 - 12 } & \multicolumn{9}{|c|}{ Bad Weather } & \multicolumn{3}{|c|}{ Average } & \multicolumn{3}{c|}{ Baseline } & Average \\
\cline { 2 - 11 } & Blizzard & Skating & SnowFall & WetSnow & & Highway & Office & Pedestrains & PETS2006 & \\
\hline ARF-MOG [17] & 0.7532 & 0.7020 & 0.7899 & 0.7154 & 0.7406 & 0.7741 & 0.3260 & 0.7120 & 0.4566 & 0.5671 \\
\hline ARF-PBAS [9] & 0.8020 & 0.7230 & 0.7555 & 0.7564 & 0.7573 & 0.8266 & 0.2855 & 0.7562 & 0.6822 & 0.6376 \\
\hline ARF-CB [12] & 0.7564 & 0.7860 & 0.7966 & 0.7852 & 0.7810 & 0.8056 & 0.7120 & 0.7751 & 0.7789 & 0.7679 \\
\hline FTSG [19] & $\mathbf{0 . 8 5 0 3}$ & $\mathbf{0 . 9 1 4 7}$ & 0.8197 & 0.7066 & 0.8228 & 0.9446 & $\mathbf{0 . 9 3 3 8}$ & $\mathbf{0 . 9 3 2 3}$ & $\mathbf{0 . 9 2 1 2}$ & $\mathbf{0 . 9 3 3 0}$ \\
\hline Bin Wang Apr [18] & 0.7177 & 0.9103 & 0.7874 & 0.6538 & 0.7673 & 0.9452 & 0.7863 & 0.9250 & 0.8688 & 0.8813 \\
\hline MSTBM Model [13] & 0.7136 & 0.5862 & 0.7141 & 0.5343 & 0.6370 & $\mathbf{0 . 9 5 3 5}$ & 0.7541 & 0.8709 & 0.8017 & 0.8450 \\
\hline Ours & 0.8496 & 0.7880 & $\mathbf{0 . 8 9 1 3}$ & $\mathbf{0 . 8 5 0 2}$ & $\mathbf{0 . 8 4 4 7}$ & 0.9166 & 0.8850 & 0.9010 & 0.8230 & 0.8814 \\
\hline
\end{tabular}

tives, and false negatives. CDnet [7] is quantitatively evaluated according to this criteria by comparing our results with available ground truth image of each sequence. However, I-LIDS dataset is evaluated according to the process described in [4] as the ground truth images are not available.

According to I-LIDS evaluation process, any alarm events presented in the first 5 minutes will be ignored and it will not effect the system performance. The detection is performed, which is compared with its ground truth data to generate the number of true positives, false positives, and false negative alarms. The detailed evaluation process can be found in [4].

Table 2. Qualitative results of CDnet [7]: Average F-measure score of each video sequence with earlier approaches.

\begin{tabular}{|c|c|c|c|c|c|c|c|}
\hline \multirow[t]{3}{*}{ Method } & \multicolumn{7}{|c|}{ Change Detection dataset [7] } \\
\hline & & & Nig & t Videos & & & Average \\
\hline & BridgeEntry & BussyBoulvard & FluidHighway & StreetCornerAtNight & TramStation & WinterStreet & \\
\hline ARF-MOG [17] & 0.3460 & 0.3792 & 0.3987 & 0.4063 & 0.3860 & 0.4420 & 0.3960 \\
\hline ARF-PBAS [9] & 0.4222 & 0.346 & 0.442 & 0.4063 & 0.412 & 0.4677 & 0.4160 \\
\hline ARF-CB [12] & 0.3445 & 0.2930 & 0.3555 & 0.3111 & 0.2890 & 0.4599 & 0.3421 \\
\hline FTSG [19] & 0.4213 & 3457 & 0.4169 & 0.5897 & 0.7017 & 0.6030 & 0.5130 \\
\hline Bin Wang Apr [18] & 0.1806 & 0.3508 & 0.1924 & 0.4971 & 0.5909 & 0.4032 & 0.3802 \\
\hline MSTBM Model [13] & 0.0256 & 0.3308 & 0.5045 & 0.2911 & 0.6443 & 0.5049 & 0.4164 \\
\hline Ours & 0.4758 & 0.3916 & 0.3575 & 0.5601 & 0.8499 & 0.4944 & 0.5215 \\
\hline
\end{tabular}

Table 1, 2, and 3 show the performance of our proposed method using CDnet [7] and I-LIDS [4] dataset as compared to other approaches. In Table 1 and 2, our method outperforms in Bad Weather and Night video sequences, as compared with the state of the art algorithms. However, a comparative performance is observed for simple cases such as Baseline category in table. 1. Moreover, the F-measure score in Table 1 and 2, depicts that we are the $4^{\text {th }}$ top best performer in Bad Weather and Night videos category, according to the online results reported in CDnet [7] website.

Time comlexity is also observed during our evaluations. The time is recorded in CPU time as $[h h: m m: s s]$ and we have $[00: 00: 56]$ for the first 100 frames 
Table 3. Qualitative results of I-LIDS dataset [4]: Average F-measure score of each video sequence with earlier approaches.

\begin{tabular}{|c|c|c|c|c|c|c|c|c|c|c|c|}
\hline \multirow{3}{*}{ Method } & \multicolumn{10}{|c|}{ I-LIDS dataset [4] } \\
\cline { 2 - 13 } & \multicolumn{10}{|c|}{ SZTRA104b } \\
\cline { 2 - 12 } & 01 & 02 & 03 & 04 & 05 & 06 & 07 & 08 & 09 & 10 & \\
\hline ARF-MOG [17] & 0.5602 & 0.7452 & 0.6022 & 0.7145 & 0.6233 & 0.4856 & 0.7566 & 0.7784 & 0.7968 & 0.7475 & 0.6856 \\
\hline ARF-PBAS [9] & 0.6032 & 0.8122 & 0.7466 & 0.7630 & 0.5820 & 0.5560 & 0.7720 & 0.7120 & 0.7502 & 0.7030 & 0.7062 \\
\hline ARF-CB [12] & 0.8030 & 0.7820 & 0.7136 & 0.7820 & 0.7030 & 0.7844 & 0.8032 & 0.8430 & 0.8530 & 0.8830 & 0.7950 \\
\hline FTSG [19] & 0.8460 & 0.8010 & 0.7563 & 0.7760 & 0.7065 & 0.7936 & 0.8230 & 0.8566 & 0.7930 & 0.8030 & 0.8125 \\
\hline Bin Wang Apr [18] & 0.6450 & 0.7720 & 0.6630 & 0.8030 & 0.6460 & 0.8530 & 0.7256 & 0.7964 & 0.7460 & 0.7974 & 0.7447 \\
\hline MSTBM Model [13] & 0.7488 & 0.8770 & 0.5633 & 0.7489 & 0.7687 & 0.7861 & $\mathbf{0 . 8 1 5 6}$ & $\mathbf{0 . 8 8 8 8}$ & 0.8654 & 0.9025 & 0.8087 \\
\hline Ours & $\mathbf{0 . 8 6 3 2}$ & $\mathbf{0 . 9 1 2 0}$ & $\mathbf{0 . 8 9 3 1}$ & $\mathbf{0 . 8 5 6 0}$ & $\mathbf{0 . 8 8 7 0}$ & $\mathbf{0 . 8 7 5 2}$ & 0.8065 & 0.8668 & $\mathbf{0 . 9 4 5 0}$ & $\mathbf{0 . 9 3 8 0}$ & $\mathbf{0 . 8 8 4 2}$ \\
\hline
\end{tabular}

having image resolution of $576 \times 720$. Since RPCA methods are not stable to process high dimensional data or they take longer time, which is not useful for real-time systems. In this study, we have achieved almost a real-time processing, in addition smoothing or suppressing the snow pixels using ARF constraints improved the background subtraction results. These good experimental evaluations are the evidence of introducing a real-time image denoising constraints together with OR-PCA.

\section{Conclusion}

In this paper, an integrated framework for improved background subtraction is presented using real time continuous constraints ARF together with OR-PCA. Basically, the proposed scheme is divided into two stages. The first part shows the robustness against intensive weather situations such as snow or rain, whereas in the second stage, where day time scene is our assumption, which is very advantageous for the night time surveillance agents to choose it for monitoring different activities. However, we just performed small evaluations on color transfer strategy due to unavailability of datasets. Therefore, our future work is mainly focus on a more robust color transfer technique which is independent of day time bright scene and this work will be further extended for moving camera case.

\section{Acknowledgments}

This work is supported by the World Class 300 project, Development of HD video/network-based video surveillance system(10040370), funded by the Ministry of Trade, Industry, and Energy (MOTIE), Korea.

\section{References}

1. Barbu, A.: Learning real-time mrf inference for image denoising. In: Computer Vision and Pattern Recognition, 2009. CVPR 2009. IEEE Conference on. pp. 15741581. IEEE (2009)

2. Barbu, A.: Training an active random field for real-time image denoising. Image Processing, IEEE Transactions on 18(11), 2451-2462 (2009) 
3. Bouwmans, T., Zahzah, E.H.: Robust PCA via Principal Component Pursuit: A review for a comparative evaluation in video surveillance. Computer Vision and Image Understanding pp. 22-34 (2014)

4. Branch, H.O.S.D.: Imagery library for intelligent detection systems I-LIDS. In: Crime and Security, 2006. The Institution of Engineering and Technology Conference on. pp. 445-448 (June 2006)

5. Candès, E.J., Li, X., Ma, Y., Wright, J.: Robust Principal Component Analysis? Journal of the ACM (JACM) 58(3), 11-37 (2011)

6. Feng, J., Xu, H., Yan, S.: Online robust PCA via stochastic optimization. In: Advances in Neural Information Processing Systems. pp. 404-412 (2013)

7. Goyette, N., Jodoin, P., Porikli, F., Konrad, J., Ishwar, P.: Changedetection.net: A new change detection benchmark dataset. In: Computer Vision and Pattern Recognition Workshops (CVPRW), IEEE Computer Society Conference on. pp. 1-8 (June 2012)

8. Hakim, A.E.A.: A novel approach for rain removal from videos using low-rank recovery. In: Proceedings of the 5th IEEE International Conference on Intelligent Systems, Modelling and Simulation. pp. 13-18 (2014)

9. Hofmann, M., Tiefenbacher, P., Rigoll, G.: Background segmentation with feedback: The pixel-based adaptive segmenter. In: Computer Vision and Pattern Recognition Workshops (CVPRW), 2012 IEEE Computer Society Conference on. pp. 38-43 (2012)

10. Hwang, Y., Lee, J.Y., Kweon, I.S., Kim, S.J.: Color transfer using probabilistic moving least squares. In: Computer Vision and Pattern Recognition (CVPR), 2014 IEEE Conference on. pp. 3342-3349. IEEE (2014)

11. Javed, S., Oh, S.H., Sobral, A., Bouwmans, T., Jung, S.K.: OR-PCA with MRF for Robust Foreground Detection in Highly Dynamic Backgrounds. In: Computer Vision-ACCV 2014, pp. 284-299. Springer (2015)

12. Kim, K., Chalidabhongse, T.H., Harwood, D., Davis, L.: Background modeling and subtraction by codebook construction. In: Image Processing, 2004. ICIP'04. 2004 International Conference on. vol. 5, pp. 3061-3064. IEEE (2004)

13. Lu, X.: A multiscale spatio-temporal background model for motion detection. In: Image Processing (ICIP), 2014 IEEE International Conference on. pp. 3268-3271. IEEE (2014)

14. Reinhard, E., Ashikhmin, M., Gooch, B., Shirley, P.: Color transfer between images. IEEE Computer graphics and applications 21(5), 34-41 (2001)

15. Roth, S., Black, M.: Fields of experts. International Journal of Computer Vision 82(2), 205-229 (2009)

16. Shah, M., Deng, J.D., Woodford, B.J.: Video background modeling: recent approaches, issues and our proposed techniques. Machine vision and applications 25(5), 1105-1119 (2014)

17. Stauffer, C., Grimson, W.E.L.: Adaptive background mixture models for real-time tracking. In: Computer Vision and Pattern Recognition, IEEE Computer Society Conference on. vol. 2. IEEE (1999)

18. Wang, B., Dudek, P.: A fast self-tuning background subtraction algorithm. In: Computer Vision and Pattern Recognition Workshops (CVPR), 2014 IEEE Conference on. pp. 401-404. IEEE (2014)

19. Wang, R., Bunyak, F., Seetharaman, G., Palaniappan, K.: Static and moving object detection using flux tensor with split gaussian models. In: Computer Vision and Pattern Recognition Workshops (CVPRW), 2014 IEEE Conference on. pp. 420424. IEEE (2014) 